\title{
LESIONES ANATOMOPATOLÓGICAS EN CUYES (Cavia porcellus) CON DIAGNÓSTICO BACTERIOLÓGICO DE Salmonella sp.
}

\author{
Anatomopathological Lesions in Guinea Pigs (CaVia porcellus) with \\ BaCteriological Diagnosis of SALMONELla SPP
}

\author{
Américo Layme M. ${ }^{1}$, Rosa Perales C. ${ }^{1,2}$, Alfonso Chavera C. ${ }^{1}$, César Gavidia C. ${ }^{3}$, \\ Sonia Calle E. ${ }^{4}$
}

\section{RESUMEN}

\begin{abstract}
El objetivo del presente estudio fue determinar la frecuencia del tipo de lesiones anatomopatológicas que predominan en órganos de cobayos infectados con Salmonella sp. Se hizo un estudio retrospectivo con 81 protocolos de necropsia del Laboratorio de Patología de la Facultad de Medicina Veterinaria de la Universidad Nacional Mayor de San Marcos, que tuvieron diagnóstico bacteriológico positivo a Salmonella sp en el periodo 2001-2007. Se clasificó las lesiones anatomopatológicas en procesos inflamatorios, trastornos circulatorios, degenerativos y de adaptación, siendo la inflamación el trastorno patológico más frecuente (177/408). Se encontró una mediana de cinco órganos afectados por animal, siendo el hígado el órgano con más lesiones patológicas $(87.7 \% \pm$ 0.1\%), donde la imagen patomorfológica predominante fue la hepatitis necrótica (36/81).
\end{abstract}

Palabras clave: cobayos, Salmonella sp, lesiones anátomopatológicas

\section{Abstract}

The aim of the study was to determine the frequency of major pathologic lesions in internal organs of guinea pigs infected with Salmonella spp. The retrospective study was conducted using 81 necropsy records from the Pathology Laboratory of the College of Veterinary Medicine, San Marcos University, which had positive bacteriological diagnosis to Salmonella spp in the period 2001-2007. Pathological lesions were classified in inflammatory processes, circulatory disorders, degenerative and adaptive, where inflammation was the most common pathological disorder (177/408). The results showed a median of five affected organs per animal, the liver being the organ with highest frequency of pathological lesions (87.7 $\pm 0.1 \%$ ), and the prevailing pathomorphological image was necrotic hepatitis (36/81).

Key words: guinea pigs, Salmonella spp, anatomopathological lesions

\footnotetext{
${ }^{1}$ Laboratorio de Histología, Embriología y Patología Veterinaria, ${ }^{3}$ Laboratorio de Medicina Veterinaria Preventiva, ${ }^{4}$ Laboratorio de Microbiología y Parasitología Veterinaria, Facultad de Medicina Veterinaria, Universidad Nacional Mayor de San Marcos, Lima

${ }^{2}$ E-mail: rperales_fmv@hotmail.com
} 


\section{INTRODUCCIÓN}

La crianza de cuyes en el Perú se desarrolla principalmente bajo la modalidad de crianza familiar; sin embargo, la crianza intensiva y comercial del cuy se ha venido incrementando en la última década, debido al aumento en la demanda de su carne, tanto en el mercado nacional como en el externo.

Se ha logrado importantes avances técnicos en la crianza a gran escala del cuy, pero los conocimientos sobre aspectos sanitarios y epidemiología de las enfermedades son aún escasos. En este contexto, destaca la salmonelosis, enfermedad que ocasiona elevada mortalidad y morbilidad en poblaciones de cuyes (Bustamante, 1993; Chauca, 1997; MINAG, 2008). La salmonelosis en el cuy es causada por serotipos del género Salmonella, bacilos gram negativos no esporulados pertenecientes a la familia Enterobacteriaceae. En el Perú, el serotipo aislado con mayor frecuencia es el serovar typhimurium, en frecuencias que superan el 95\% en relación a otros serotipos (Ameghino, 1968; Ramírez, 1972; Bustamante, 1993; Garmendia et al., 2000).

Los alimentos contaminados son la principal fuente de contagio; sin embargo, la introducción de animales con antecedentes sanitarios desconocidos es otra de las fuentes de contagio de importancia. La enfermedad, por su carácter transmisible, se disemina rápidamente en la población expuesta, la que asociada a alteraciones del medio ambiente, tiene un curso epidémico inicial y un comportamiento endémico posterior (Ramírez, 1972; Pérez, 1975; Stellmacher, 1981; Jubb et al., 1990; Radostits et al., 2002).

La salmonelosis en cuyes se manifiesta en una forma aguda y una forma crónica. La primera se presenta como un cuadro septicémico agudo, donde la muerte ocurre en un lapso de 24 a 48 horas, en muchos casos, sin mostrar signo clínico alguno, aunque en otras ocasiones se observa decaimiento, postración, anorexia, opistótono, parálisis de los miembros posteriores, diarrea con moco, y aborto. En los casos crónicos, es notorio un adelgazamiento paulatino, pelaje deslucido y aumento del volumen abdominal (Ramírez, 1972; Bustamante, 1993; Evans, 2005). Dentro de las alteraciones patológicas se encuentra la afección de múltiples órganos con procesos congestivos e inflamatorios (Ameghino, 1968; Ramírez, 1972).

El objetivo del estudio fue contribuir en el conocimiento de las lesiones anatomopatológicas que origina la salmonelosis en cuyes, al hacer un análisis retrospectivo de los hallazgos patológicos en cuyes con diagnóstico de salmonelosis.

\section{Materiales y Métodos}

El estudio retrospectivo se llevó a cabo en la Facultad de Medicina Veterinaria de la Universidad Nacional Mayor de San Marcos, Lima. Se trabajó con los protocolos de necropsia de cuyes con historia de salmonelosis del Laboratorio de Patología y los registros de aislamiento bacteriológico de Salmonella sp en el Laboratorio de Bacteriología ocurridos en el periodo comprendido entre 2001 al 2007.

Se registró la siguiente información: aislamiento de Salmonella sp (positivo, negativo), sexo del animal (macho, hembra), edad [lactante (nacimiento a tres semanas), recría (de tres semanas a tres meses), adulto (mayor a tres meses)], lugar de procedencia (Lima Norte, Lima Sur, Lima Centro, Lima Este, Callao, otros), órgano lesionado, y tipo de lesión (inflamación, trastorno circulatorio, degeneración, adaptación, aumento inespecífico de tamaño o megalia).

Se determinó la frecuencia de órganos lesionados y el proceso patológico que presentaron los cuyes afectados con salmonelosis. 
Cuadro 1. Órganos y tipo de lesiones anatomopatológicas en 81 cuyes positivos a Salmonella sp (2001-2007)

\begin{tabular}{|c|c|c|c|c|c|c|}
\hline & \multicolumn{5}{|c|}{ Órganos } & \multirow{2}{*}{ Total } \\
\hline & Hígado & Intestino & Pulmón & Bazo & Otros & \\
\hline Inflamación & 53 & 40 & 30 & 4 & 50 & 177 \\
\hline $\begin{array}{l}\text { Trastorno } \\
\text { circulatorio }\end{array}$ & 10 & 14 & 14 & 7 & 97 & 142 \\
\hline Degeneración & 8 & 0 & 3 & 0 & 35 & 46 \\
\hline Adaptación & 0 & 0 & 0 & 6 & 0 & 6 \\
\hline $\begin{array}{l}\text { Aumento } \\
\text { inespecífico de } \\
\text { tamaño }\end{array}$ & 0 & 0 & 0 & 25 & 12 & 37 \\
\hline N. ${ }^{o}$ de órganos & 71 & 54 & 47 & 42 & 194 & 408 \\
\hline$\% \pm \mathrm{IC}^{1}$ & $87.7 \pm 0.1$ & $66.7 \pm 0.1$ & $58.0 \pm 0.1$ & $51.9 \pm 0.1$ & & \\
\hline
\end{tabular}

${ }^{1}$ Intervalo de confianza del $95 \%$

Cuadro 2. Órganos lesionados, según el tipo de exudado inflamatorio, en 81 cuyes positivos a Salmonella sp (2001-2007)

\begin{tabular}{lcccccc}
\hline & Hígado & Intestino & Pulmón & Bazo & Otros & $\begin{array}{c}\text { Total de } \\
\text { órganos }\end{array}$ \\
\hline Catarral & 0 & 18 & 0 & 0 & 3 & 21 \\
Hemorrágica & 0 & 9 & 22 & 0 & 17 & 48 \\
Necrótica & 36 & 6 & 5 & 1 & 8 & 56 \\
Fibrinosa & 10 & 0 & 2 & 1 & 6 & 19 \\
Serosa & 0 & 5 & 1 & 0 & 5 & 11 \\
Ulcerosa & 0 & 0 & 0 & 0 & 4 & 4 \\
Erosiva & 0 & 0 & 0 & 0 & 1 & 1 \\
Purulenta & 7 & 2 & 0 & 2 & 6 & 17 \\
\hline \multicolumn{1}{c}{ N. ${ }^{\circ}$} & 53 & 40 & 30 & 4 & 50 & 177 \\
\multicolumn{1}{c}{$\%$ IC } & $65.4 \pm 0.1$ & $49.4 \pm 0.1$ & $37.0 \pm 0.1$ & $4.9 \pm 0.1$ & & \\
\hline
\end{tabular}

${ }^{1}$ Intervalo de confianza del 95\%

\section{Resultados}

En el periodo 2001-2007 se realizaron 125 necropsias en cuyes con diagnóstico presuntivo de salmonelosis. De estos, el 64.8 $\pm 8.4 \%(81 / 125)$ fue positivo al aislamiento de Salmonella sp. Se trabajó con los 81 protocolos de necropsia con diagnóstico bacteriológico positivo. 
Cuadro 3. Órganos lesionados, según el tipo de trastorno circulatorio, en 81 cuyes positivos a Salmonella sp (2001-2007)

\begin{tabular}{lcccccccc}
\hline & $\begin{array}{c}\text { Cavidad } \\
\text { abdominal }\end{array}$ & Riñón & Útero & Intestino & Pulmón & $\begin{array}{c}\text { Saco } \\
\text { pericár- } \\
\text { dico }\end{array}$ & Otros & $\begin{array}{c}\text { Total } \\
\text { de } \\
\text { órganos }\end{array}$ \\
\hline Edema & 29 & 0 & 0 & 0 & 0 & 13 & 5 & 47 \\
Congestión & 0 & 15 & 8 & 13 & 6 & 0 & 20 & 62 \\
Hemorragia & 0 & 7 & 7 & 1 & 8 & 1 & 8 & 32 \\
Infarto & 0 & 0 & 0 & 0 & 0 & 0 & 1 & 1 \\
\hline N. & 29 & 22 & 15 & 14 & 14 & 14 & 34 & 142 \\
$\%$ & 35.8 & 27.2 & 18.5 & 17.3 & 17.3 & 17.3 & & \\
\hline
\end{tabular}

${ }^{1}$ Intervalo de confianza del 95\%

Cuadro 4. Órganos afectados, según el tipo de degeneración, en 81 cuyes positivos a Salmonella sp (2001-2007)

\begin{tabular}{llcc}
\hline \multirow{2}{*}{ Órgano } & Degeneración & \multicolumn{2}{c}{ Total } \\
\cline { 3 - 4 } & & N. ${ }^{\circ}$ & $\% \pm$ IC $^{1}$ \\
\hline \multirow{2}{*}{ Hígado } & Degeneración hidrópica & 5 & $6.2 \pm 0.05$ \\
\multirow{2}{*}{ Pulmón } & Degeneración grasa & 3 & $3.7 \pm 0.04$ \\
\multirow{2}{*}{ Vesícula biliar } & Enfisema & 2 & $2.5 \pm 0.03$ \\
\multirow{2}{*}{ Riñón } & Antracosis & 1 & $1.2 \pm 0.02$ \\
& Dilatación & 29 & $34.6 \pm 0.10$ \\
\hline Total de órganos & Retracción & 6 & $7.4 \pm 0.06$ \\
\hline
\end{tabular}

${ }^{1}$ Intervalo de confianza del 95\%

El 2.8, 30.2 y $67.0 \%$ estuvo en la etapa de lactancia, recría y adulto, respectivamente, y el $86.8 \%$ fue macho (59/68). No estuvo registrado el sexo o la edad en 13 casos. Asimismo, la mayoría de cobayos procedieron de Lima sur (40.3\%) y Lima este (33.9\%), aunque no se pudo obtener la información respectiva en 19 casos.
Las lesiones anatomopatológicas se observaron en la mayoría de órganos, con una mediana de 5 órganos por animal. El hígado fue el órgano con mayor frecuencia de lesiones (87.7\%), seguido por el intestino, pulmón y bazo (Cuadro 1).

El tipo de lesión más frecuente fue la inflamación del hígado (65.4\% del total de 


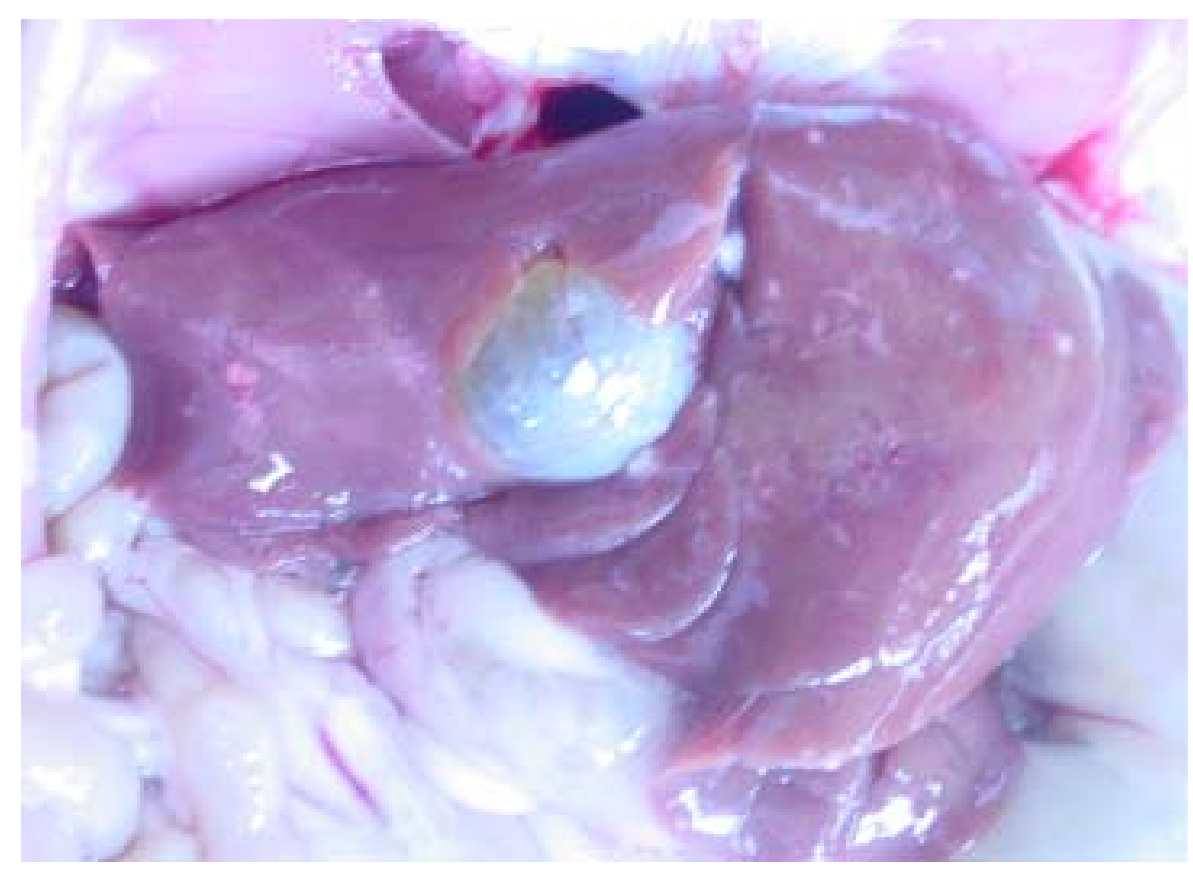

Figura 1. Hepatitis necrótica multifocal en un cuy positivo a Salmonella sp. Nótese los focos necróticos en la superficie del parénquima hepático y también la dilatación de la vesícula biliar

cuyes); asimismo, el tipo de exudado más común fue el necrótico, localizándose mayormente en el hígado (Fig. 1), representando el $67.9 \%(36 / 53)$ de los cobayos con proceso inflamatorio en hígado (Cuadro 2).

El Cuadro 3 muestra el tipo de trastornos circulatorios en los órganos afectados, donde predominó el edema de la cavidad abdominal (ascitis), seguido por la congestión renal e intestinal y edema del saco pericárdico (hidropericardio). El Cuadro 4 muestra los procesos degenerativos que se encontraron en 46 órganos, siendo la dilatación de la vesícula biliar la de mayor predominio (34.6 $\pm 0.1 \%$ ).

Las lesiones anatomopatológicas se observaron en la mayoría de órganos, con una mediana de 5 órganos por animal. El hígado fue el órgano con mayor frecuencia de lesiones (87.7\%), seguido por el intestino, pulmón y bazo (Cuadro 1).
Lesiones de adaptación se encontraron únicamente en el bazo, donde se presentó hiperplasia folicular linfoide en seis animales (7.4 $\pm 0.06 \%)$. Aumento de tamaño (megalia) se encontró en bazo en 25 cuyes (30.9 \pm $0.07 \%$ ), en ganglio mesentérico en 11 cuyes (13.6 $\pm 0.08 \%)$ y un caso en riñón (1/81).

El órgano con mayor frecuencia de aislamiento fue el hígado (24.1 $\pm 0.05 \%, 68 / 282)$, seguido por el bazo, intestino, útero pulmón y vesícula biliar.

\section{Discusión}

Cuyes infectados con Salmonella sp presentaron diversos órganos afectados, evidenciando el carácter septicémico en esta especie (Ameghino, 1968; Ramírez, 1972; Morales et al., 1995), al igual que ocurre en 
otras especies, como cerdos y humanos, con Salmonella choleraesuis y Salmonella typhi, respectivamente (Martínez, 2007).

El predominio de lesiones en el hígado (Ramírez, 1972) y bazo (Nelson y Smith, 1927) puede deberse al tropismo de la bacteria hacia los órganos linfoides. Una vez que la Salmonella llega al lumen intestinal, invade al hospedero a través de enterocitos y células $\mathrm{M}$, células relacionadas con los nódulos linfáticos de las placas de Peyer del íleon, para ser transportadas por macrófagos, vía linfática al hígado y bazo. Además, se ha demostrado una vía alterna, en la cual, fagocitos CD 18 transportan la bacteria directamente del lumen intestinal a la circulación, bazo e hígado. De esta manera, el hígado y el bazo pueden ser colonizadas sin ocasionar daño intestinal (Figueroa y Verdugo, 2005). Una vez establecida la infección sistémica, la salmonelosis puede desarrollarse como enfermedad, ocasionando septicemia y localización en diversos tejidos (Jubb et al., 1990; Radostits et al., 2002; Santos et al., 2003; Figueroa y Verdugo, 2005).

La hepatitis necrótica, expresión patomorfológica más frecuente del proceso inflamatorio encontrado en el presente estudio, confirma los reportes de otros autores (Ramírez, 1972; Morales et al., 1995). La necrosis hepática focal es una imagen macroscópica común en infecciones bacterianas septicémicas en otras especies. $\mathrm{Al}$ parecer, la necrosis focal puede ser el resultado de una reacción de las células de Kupffer que liberan citocinas ante el estímulo de endotoxinas o gérmenes (Jubb et al., 1990; Diago y Huguet, 2006).

El intestino fue el segundo órgano con mayor frecuencia de lesiones, especialmente de tipo inflamatorio. La lesiones en intestino son comprensibles ya que la mucosa intestinal constituye la puerta de entrada para la infección generalizada. Salmonella enterica invade enterocitos y células $\mathrm{M}$ mediante la actuación de un sistema de secreción de tipo 3 (SST3), codificada por la isla de patogenicidad SPI 1. Además, la actuación de este sistema inicia la liberación por parte de las células epiteliales de Citoquinas CXC, como la interleucina 8 (IL8), que ejerce una acción quimiotáctica sobre polimorfonucleares neutrófilos (PMN), importantes agentes defensivos de la respuesta inmune inespecífica del hospedador (Tükel et al., 2006). El incremento de la permeabilidad vascular que acompaña a la inflamación en combinación con la pérdida de la integridad epitelial de la mucosa intestinal provoca la diarrea (Figueroa y Verdugo, 2005).

La neumonía tipo hemorrágica fue la imagen patomorfológica más frecuente en pulmón, tal y como ha sido demostrada en otros estudios (Ramírez, 1972; Onyekaba, 1983; Morales et al., 1995). Procesos neumónicos ocasionados por salmonelosis se pueden observar en otras especies, sobre todo del tipo crónico en cerdos y bovinos (Radostits et al., 2002). El exudado hemorrágico se observa en infecciones sistémicas, como las causadas por salmonelosis, posiblemente debido a la liberación de toxinas por parte de la bacteria, que generan vasculitis pulmonar aguda (capilaritis), produciéndose el aumento de la permeabilidad capilar y la consiguiente salida de eritrocitos al espacio extracelular por diapédesis (Gazquez, 1991; Novoa et al., 2006).

Esplenomegalia se presentó en 25 animales, sin llegarse a determinar el proceso patológico que ocasionó el aumento de tamaño; anomalía que se ha reportado en cuyes y otras especies (Ramírez, 1972; Onyekaba, 1983, 1985; Morales et al., 1995; Ruiz et al., 2008). Sin embargo, los procesos inflamatorios y trastornos circulatorios como edema y congestión, y los cambios adaptativos como la hiperplasia linfoide, pueden estar implicados en el aumento de tamaño, no sólo del bazo sino de cualquier otro órgano. 
La vesícula biliar es un órgano muy pocas veces tomado en cuenta en el diagnóstico presuntivo de la salmonelosis; sin embargo, en el presente estudio se encontró que la dilatación de la vesícula biliar fue la tercera lesión anatomopatológica en términos de frecuencia.

Lesiones como linfadenomegalia y la metritis se encontraron con menor frecuencia en el presente estudio; en tanto que Onyekaba (1983) las destaca como lesiones de importancia en casos de salmonelosis en cuyes, de allí que deberían tenerse en cuenta durante en desarrollo de las necropsias.

El aislamiento mayoritario de la bacteria de muestras de hígado y bazo concuerda con otros resultados en la literatura (Ramírez, 1972; Matsuura, 2008).

\section{Conclusiones}

- Las lesiones anatomopatológicas se evidenciaron en diversos órganos, corroborando el proceso séptico de la salmonelosis en el cuy.

- El hígado fue el órgano donde se aisló con mayor frecuencia Salmonella sp y el órgano con mayor frecuencia de lesiones anatomopatológicas, especialmente la hepatitis necrótica.

\section{Literatura Citada}

1. Ameghino EF. 1968. Sobre un brote de salmonelosis en cuyes (Cavia cobaya): $3^{\text {er }}$ Boletín Extraordinario. Lima: IVITA. p 260-261.

2. Bustamante J. 1993. Producción de cuyes. Lima: Universidad Nacional Mayor de San Marcos. 259 p.

3. Chauca L. 1997. Producción de cuyes (Cavia porcellus). Roma: Organización de las Naciones Unidas para la Agricultura y la Alimentación - FAO. 78 p.
4. Diago M, Huguet M. 2006. Enfermedades hepáticas infecciosas. [Internet], [Octubre 2006]. Disponible en: http:// www.ghcontinuada.com/contenidos/pdf/ v5n5a353pdf001.pdf

5. Evans AR. 2005. Import risk analysis: Domestic guinea pig, Cavia porcellus, imported from Australia [Internet], [23 octubre 2007]. Disponible en: http:/ hin t link . com / g u ine a_pig / Nzriskanalysis.pdf

6. Figueroa I, Verdugo A. 2005. Месаnismos moleculares de patogenicidad de Salmonella sp. Rev Latinoam Microbiol 47: 25-42.

7. Garmendia MM, Selgrad S, Alezones F. 2000. Salmonelosis en animales de laboratorio. FONAIAP [Internet], [Noviembre 2000]. Disponible en: http:// w w w . c e n i a p.g o v. ve / p b d / RevistasTecnicas/FonaiapDivulga/fd68/ texto/mgarmendia.htm

8. Gazquez OA. 1991. Patología Veterinaria. Madrid: McGraw-Hill Interamericana. $305 \mathrm{p}$.

9. Jubb KV, Kennedy PC, Palmer N. 1990. Patología de los animales domésticos. $3^{\text {a }}$ ed. Montevideo: Agropecuaria Hemisferio Sur. $160 \mathrm{p}$.

10. Martínez AN. 2007. Virulencia, resistencia y elementos genéticos móviles en serotipos no prevalentes de Salmonella enterica. Tesis de Doctor. España: Universidad de Oviedo. 202 p.

11. Matsuura SA. 2008. Susceptibilidad a antibacterianos in vitro de Salmonella enterica aislada de cobayos de crianza familiar-comercial en la provincia de Carhuaz. Tesis de Médico Veterinario. Lima: Univ Nacional Mayor de San Marcos. 65 p.

12. [MINAG] Ministerio de Agricultura. 2008. Lima: MINAG. [Internet], [Octubre 2008]. Disponible en: http:// www.minag.gob.pe/situacion-de-las-actividades-de-crianza-y-produccion/ cuyes-39.html 
13. Morales CM, Hung A, Alvarado A. 1995. Mortalidad por salmonelosis en cobayos. En: Investigaciones en cuyes. APPA 1994-2007. [Internet]. Disponible en: http://www.inia.gob.pe/documentos/ APPA-RESUMEN-1994-2007.pdf

14. Nelson J, Smith T. 1927. Report of a natural outbreak of paratyphoid in a guinea pig population. J Exp Med 45: 353363.

15. Novoa LA, Conde F, Guardiola B, Rodríguez C, Navia R. 2006. El reto de las neumonías hemorrágicas [Internet]. Disponible en: http:// bvs.sld.cu/revistas/gme/pub/ vol.8.\%283\%29_09/p9.html

16. Onyekaba CO. 1983. Clinical salmonellosis in a guinea pig colony caused by a new Salmonella serotype, Salmonella ochiogu. Lab Anim 17: 213216.

17. Onyekaba CO. 1985. Salmonella ochiogu: experimental in of laboratory rats (Rattus rattus). Lab Anim 19: 148151.

18. Pérez Z. 1975. Investigación de salmonelas en cobayos (Cavia porcellus) aparentemente normales. Tesis de Biología. Lima: Univ Nacional Mayor de San Marcos. 18 p.
19. Radostits OM, Gay CC, Blood DC, Hinchcliff KW. 2002. Tratado de las enfermedades del ganado bovino, ovino, porcino, caprino, y equino. $9^{a}$ ed. Madrid: Mc Graw-Hill. 959 p.

20. Ramírez I. 1972. Estudio bacteriológico y epidemiológico de un brote infeccioso en cobayos (Cavia porcellus). Tesis de Médico Veterinario. Lima: Univ Nacional Mayor de San Marcos. 62 p.

21. Ruiz G, Constantino F, Quintana J, Cedillo C, Urquiza O. 2008. Patogenia de Salmonella enteritidis FT $13^{\mathrm{a}} \mathrm{y}$ Salmonella enteritidis biovar Issatschenko en pollos de engorda. Vet Mex 39: 145-160.

22. Santos R, Tsolis R, Bäumler A, Adams I. 2003. Patogénesis of salmonellainduced enteritis. Braz J Med Biol Res 36: 3-12.

23. Stellmacher W. 1981. Infecciones por Salmonellas. En: Beer J (ed). Enfermedades infecciosas de los animales domésticos. Zaragoza: Acribia. p 59-81.

24. Tükel C, Raffatellu M, Chessa D, Wilson RP, Akcelik M, Bäumler AJ. 2006. Neutrophil influx during nontyphoidal salmonellosis: Who is in the drivers seat? FEMS Immunol Med Microbiol 46: 320-329. 